\title{
More on Tanaka-Tagoshi Parametrization of post-1PN Spin-Free Gravitational Wave Chirps: Equispaced and Cardinal Interpolated Lattices
}

\author{
R.P. Croce and Th. Demma \\ Wavesgroup, D.I. ${ }^{3}$ E., University of Salerno, Italy \\ V. Pierro and I.M. Pinto \\ Wavesgroup, University of Sannio at Benevento, Italy
}

(Dated: November 1, 2018)

\begin{abstract}
The conclusions obtained in gr-qc/0101067 are shown to be valid also if the full 2.5PN expansion of the chirp phase is used.

PACS numbers: 04.80.Nn, 95.55.Ym, 95.75.Pq, 97.80.Af
\end{abstract}

In a recent paper [1] we shew that the spin-free gravitational wave templates parametrization introduced by Tanaka and Tagoshi in 22 is effective to set up uniformly spaced post-1PN template lattices subject to a given minimal match constraint. Lattice-uniformity makes cardinal interpolation of the match [3], 任] in the $2 D$ Tagoshi-Tanaka parameter space straightforward, yielding a reduction in the $2 \mathrm{PN}$ template density and total number by a factor $\sim 4$ at $\Gamma=0.97$.

In [1] the 2PN approximant of the (spectral, spin-free) chirp phase has been used. On the other hand, in [2] the 2.5PN phase (originated in (5)) has been used, which contains (besides an irrelevant $f$-independent term, which is absorbed in the unknown coalescency phase $\phi_{C}$ ) the additional term:

$$
\frac{\pi}{128 \eta}\left(\frac{38645}{252}+5 \eta\right) \cdot \log \left(f / f_{0}\right)
$$

where $\eta=m_{1} m_{2} / m^{2}$ is the symmetric mass-ratio, and $f_{0}$ is a suitable scaling frequency [6]. It has been pointed out [5] that the $2.5 \mathrm{PN}$ phase agrees, in the limit $\eta \rightarrow 0$, with the one obtained by Tagoshi and Sasaki in the (perturbative) test mass-limit solution of the spin-free relativistic two-body problem [7]. While this might be a good reason to include the $2.5 \mathrm{PN}$ term (0.1) in the template phase, it does not result into better overlaps with exact (numerically computed) waveforms. Indeed, as shown in [8], 2.5PN templates yield generally poorer overlaps (and larger biases) as compared to $2 \mathrm{PN}$, in view of the peculiar (oscillating) behaviour of standard PN approximants [8].

However, the conceptual question is posed whether the results in [1] still hold if the 2.5PN logarithmic term (0.1) is included in the template phase. The purpose of this note is to show that the main conclusions in [1] do apply in this case too, though a number of differences are noted, which are summarized below.

The major difference, after the inclusion of the $2.5 \mathrm{PN}$ logarithmic term (0.1) in the phase, is a moderate increase in the area of the Tanaka-Tagoshi (flattened) manifold $\mathcal{T}$, yielding a correspondingly larger number of templates, as seen by comparing Fig. 1 and Table I of this note to Fig. 3 and Table III of [1], respectively.

The gaussian curvature of the spin-free manifold retains the same order of magnitude, as seen by comparing Fig. 2 of this note to Fig.s 1 and 2 of [1].

The main relevant conclusions in [1] do not change. As shown in [1], the minimal match degradation due to using the (flattened) Tanaka-Tagoshi (spin-free) parameter-space manifold in place of the (curved) true one, can be gauged in terms of the quantity $\eta$ defined in Eq. (III.6 ) of [1]. Even using the 2.5PN phase, the quantity $\eta$ is still of the same order of magnitude as the corresponding one computed in [1]. This is seen by comparing Fig. 3 of this note to Fig.s 5 and 6 of [1].

\section{Acknowledgements}

The Authors thank dr. A. Pai (IUCAA) for having posed the problem, and dr. B. Owen (Univ. of Wisconsin Milwaukee) for pinpointing an error in the first version of this note.

[1] R.P. Croce, Th. Demma, V. Pierro and I.M. Pinto, gr-qc/0101067; in print on Phys. Rev. D63 (2001).

[2] T. Tanaka and H. Tagoshi, Phys. Rev. D62, 082001 (2000).

[3] R.P. Croce, Th. Demma, V. Pierro and I.M. Pinto, Phys. Rev. D62, 124020 (2000). 
[4] R.P. Croce, Th. Demma, V. Pierro, I.M. Pinto, D. Churches and B.S. Sathyaprakash, Phys. Rev. D62, 121101(R) (2000).

[5] L. Blanchet, T. Damour, B. Iyer, C.M. Will and A. Wiseman, Phys. Rev. Lett., 74, 3515 (1995).

[6] Scaling $f$ to $f_{0}$ in the argument of (0.1) entails an irrelevant constant additive phase which is absorbed in the unknown coalescency phase $\phi_{C}$.

[7] H. Tagoshi and M. Sasaki, Progr. Th. Phys., 92, 745 (1994).

[8] T. Damour, B. Iyer and B.S. Sathyaprakash, Phys. Rev. D57, 885 (1998).

\begin{tabular}{|c|c|c|}
\hline \hline Antenna & Simplex area $\left[\mathrm{sec}^{2}\right]$ & No. of templates at $\Gamma=0.97$ \\
\hline TAMA300 & 10266 & $1.71 \cdot 10^{5}$ \\
\hline GEO600 & 57093 & $9.51 \cdot 10^{5}$ \\
\hline LIGO-I & 24732 & $4.12 \cdot 10^{5}$ \\
\hline VIRGO & 776392 & $1.29 \cdot 10^{7}$ \\
\hline \hline
\end{tabular}

Table I - 2.5PN flat simplex area and number of templates at $\Gamma=.97$, for $0.2 M_{\odot} \leq m_{1} \leq m_{2} \leq 10 M_{\odot}$. 

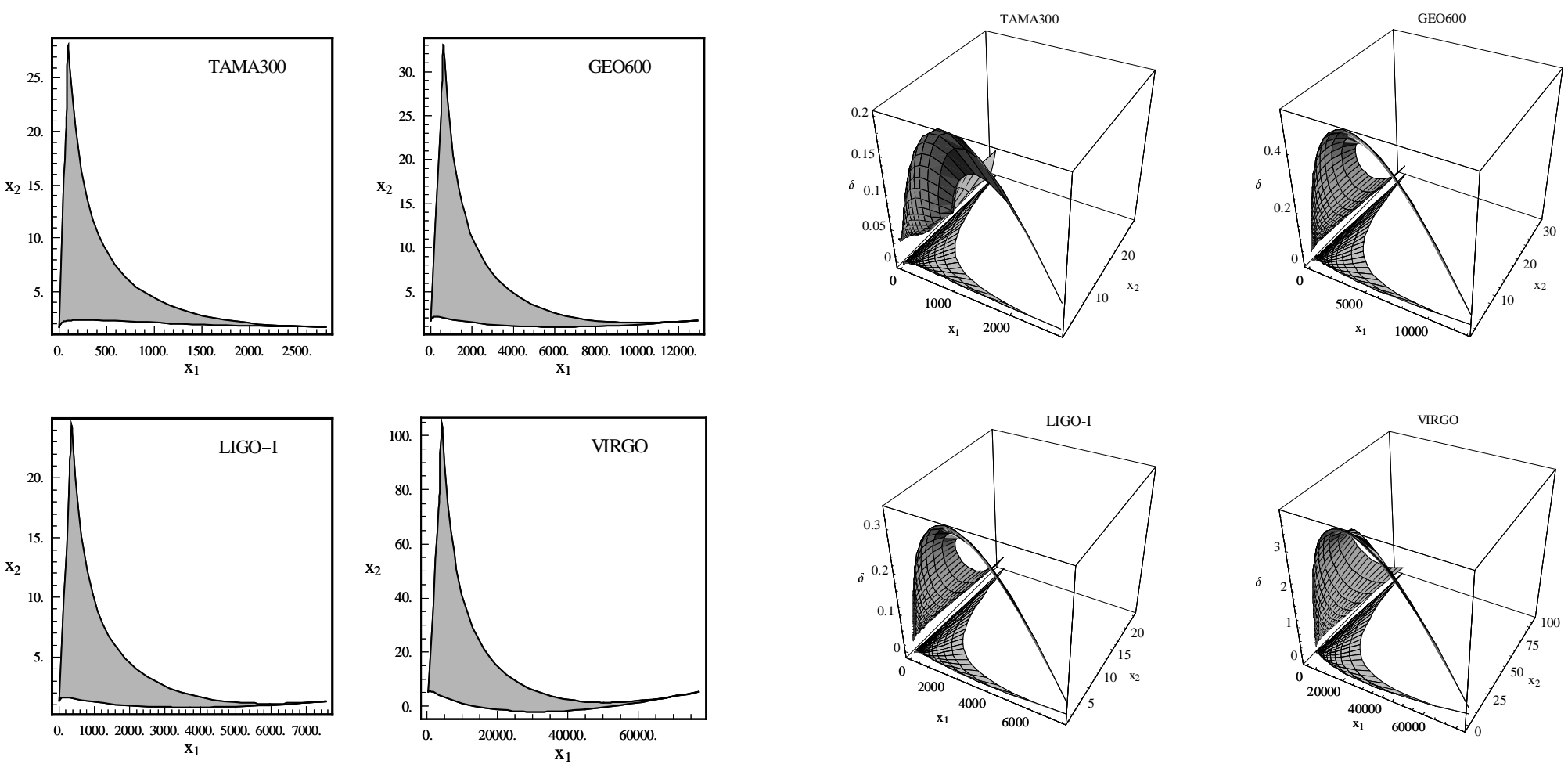

Fig 1 - Left: the 2.5PN flat simplexes corresponding to $0.2 \mathrm{M}_{\odot} \leq \mathrm{m}_{1} \leq \mathrm{m}_{2} \leq 10 \mathrm{M}_{\odot}$ for TAMA300, GEO600, LIGO-1 and VIRGO; right: the euclidean distance between the 2.5PN manifolds $\mathcal{P}$ and $\mathcal{T}$ as a function of $\left(\mathrm{x}_{1}, \mathrm{x}_{2}\right)$ for TAMA300, GEO600, LIGO-I and VIRGO. 

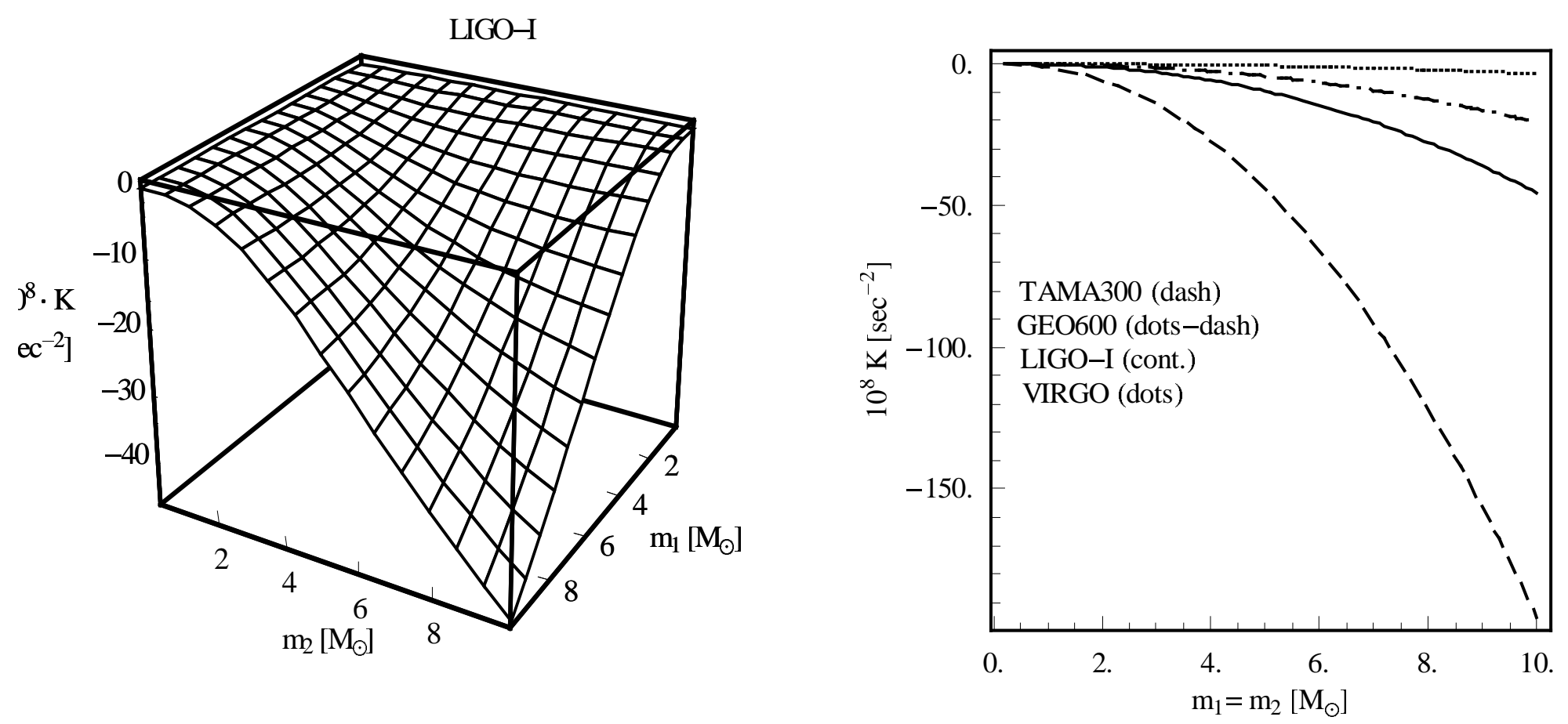

Fig. 2 - Left: gaussian curvature $\mathrm{K}\left[\mathrm{sec}^{-2}\right]$ of spin-free 2.5PN parameter space manifold vs. $\mathrm{m}_{1}, \mathrm{~m}_{2}$ for LIGO-1; right: the same quantity vs. $\mathrm{m}_{1}=\mathrm{m}_{2}$ (worst case) for TAMA300, GEO600, LIGO-1 and VIRGO. 

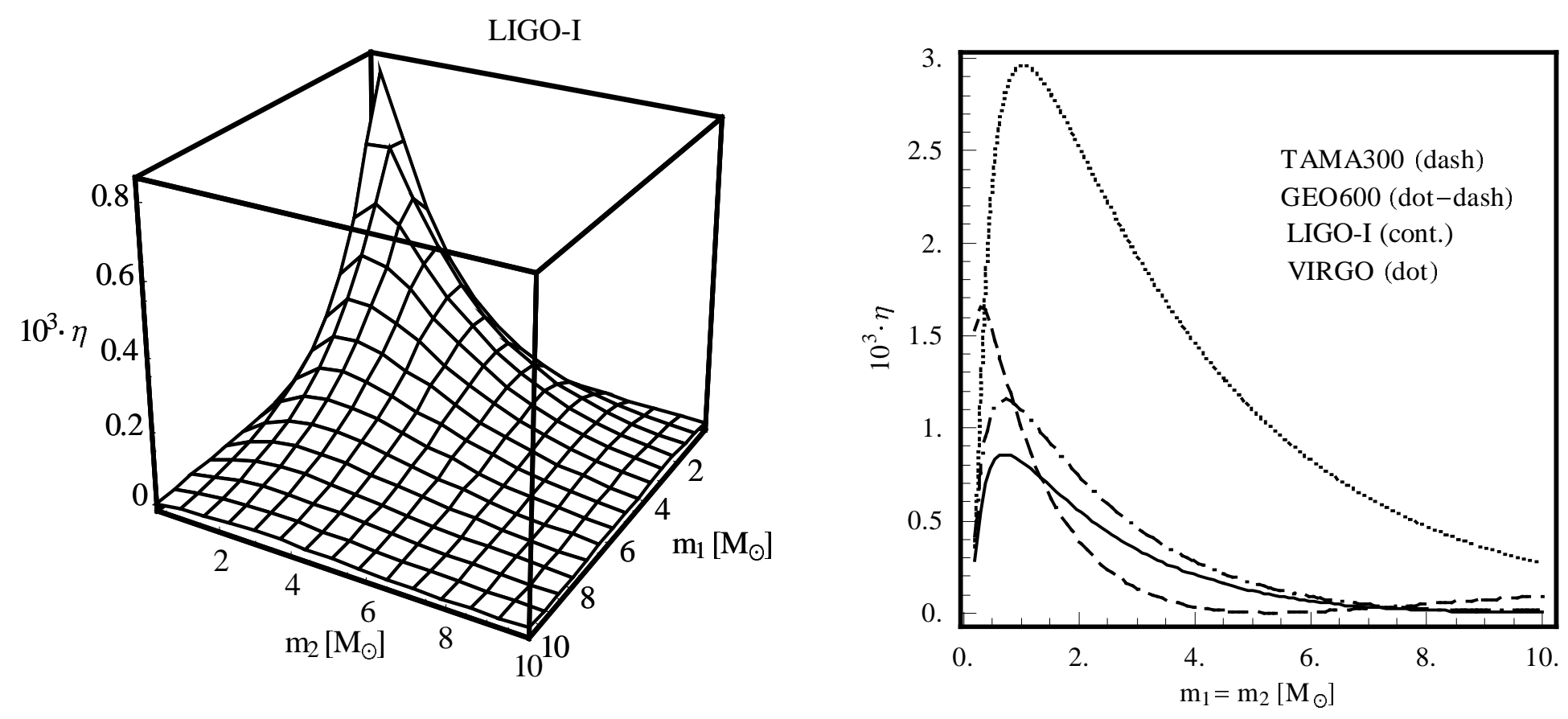

Fig 3 - Left: the 2.5PN quantity $\eta$ (eq.III.6 of [1]) vs. $\mathrm{m}_{1}, \mathrm{~m}_{2}$ for LIGO-I; right: the same quantity vs. $\mathrm{m}_{1}=\mathrm{m}_{2}$ (worst case) for TAMA300, GEO600, LIGO-I and VIRGO. 